\section{Transmission of Branch-inducing Phytoplasma PoiBI from Poinsettia (Euphorbia pulcherrima) to Crown-of-thorns (E. milii)}

\section{Mogens Nicolaisen ${ }^{1}$}

Department of Crop Protection, Danish Institute of Agricultural Sciences, Slagelse, DK 4200, Denmark

Additional index words. free branching, phytoplasma, Euphorbia

Abstract. Phytoplasma PoiBI is responsible for free branching in poinsettia (Euphorbia pulcherrima Wild. ex. Klotzsch). In this study, PoiBI was transmitted by dodder from poinsettia to crown-of-thorns ( $E$. milii Des Moulins) in one out of $\approx 100$ attempts, whereas grafting transmission was unsuccessful. PoiBI was shown to be viable in $E$. milii, as it was detected in the recipient plant and in its cuttings over 1.5 years by polymerase chain reaction. It was shown that PoiBI induces free branching in $E$. milii as well as in poinsettia. Smaller leaves, reduced growth rates, and delayed flowering were other effects of PoiBI infection.

In 1997, a phytoplasma was identified as the causal agent of the desirable and economically important free branching in poinsettia (Lee et al., 1997). The phytoplasma was characterized using polymerase chain reaction restriction fragment-length polymorphism (PCR-RFLP) analysis and the predominant strain present was shown to belong to the $16 \mathrm{~S}$ rDNA group III, which includes X-disease and related phytoplasmas (Lee et al., 1997). This branchinducing phytoplasma has been named PoiBI (Lee et al., 1998).

Free branching in ornamentals is a desirable trait for large-scale production of cuttings and, more importantly, for the production of marketable plants. Therefore, it may be desirable to transmit PoiBI into other ornamentals to increase branching. Preil and Ebbinghaus (1998) were able to transmit PoiBI into E. fulgens Karw. (scarlet plume) by grafting, resulting in significantly increased branching.

The present study aimed to investigate the host range of PoiBI by attempting to infect $E$. milii (crown-of-thorns) to investigate the effect on branching and to determine other characteristics of PoiBI infection in E. milii.

\section{Materials and Methods}

Plant material and growth conditions. Plants of 'Lilo' poinsettia were obtained in which PoiBI was present but poinsettia mosaic virus (PnMV) was absent (Bech and Rasmussen, 1996). Absence of PnMV was confirmed by ELISA using polyclonal antibodies produced at the Danish Institute of Agricultural Sciences (DIAS, Slagelse, Denmark). These plants were used as donors in transmission experiments. Eight cultivars of E. milii were

Received for publication 30 Dec. 2003. Accepted for publication 10 July 2003. The author thanks Gartneriet Egegaard and the Botanical Garden, University of Copenhagen for providing plant material.

${ }^{1}$ E-mail mogens.nicolaisen@ agrsci.dk. provided by Gartneriet Egegaard (Halk, Denmark), and plants with no detectable level of PnMV in ELISA tests were selected for the transmission experiments. These plants were further tested for phytoplasma by PCR and found not to contain any detectable level of phytoplasma.

Plants were grown at $20 / 18{ }^{\circ} \mathrm{C}$ day/night and a daylength of $15 \mathrm{~h}$ in glasshouses under insect-proof conditions using screens not penetratable by aphids. Plants were irrigated with a nutrient solution according to good practice (Ecke et al., 1990).

Transmission by grafting or dodder. Grafting between PoiBI-infected poinsettia (donor) and $E$. milii (recipient) was done either by approach grafting or by grafting $E$. milii on poinsettia rootstocks. After $\approx 3$ months, E. milii was removed from the poinsettia rootstock and rooted.

Dodder (Cuscuta reflexa Roxb.) was obtained from the Botanical Garden, University of Copenhagen, Denmark, and the absence of phytoplasma was confirmed by PCR. It was maintained on Geranium odoratissimum L. and, from there, established on poinsettia. Several months after establishment on poinsettia, dodder was established on E. milii while still maintaining the contact to poinsettia. Dodder contact was maintained for a minimum of 6 months before testing E. milii for PoiBI by PCR.

PCR test for PoiBI. Using 2 ul of genomic DNA extracted from E. milii according to Zhu et al. (1993), nested PCR was performed using the method of Abad et al. (1997). Ten microliters of each nested PCR reaction were analyzed by electrophoresis in a $1 \%(\mathrm{w} / \mathrm{v})$ agarose gel stained with ethidium bromide. PCR products from positive samples, together with samples from poinsettia, were cloned and sequenced using standard methods.

\section{Results and Discussion}

About 100 grafts were made with PoiBI- infected 'Lilo' poinsettia as rootstock and $E$. milli as scion, and $\approx 100$ connections were made between E. pulcherrima and E. milii using dodder. The grafts were established for a minimum of 3 months, although E. milii scions were actually growing during this period in only a few cases. After 3 months, the scions from the grafting experiments were detached and rooted and, after 6 months, the recipient E. milii from the dodder experiments were detached. The recipient plants were tested by PCR for the presence of PoiBI 1 month after detachment and again after 6 months. After 1 month, PCR tests showed that two scions of the grafted plants (E. milii 'Copperred' and 'Sunshine') and one plant (E. milii 'Butterfly') derived from the dodder experiments were infected with PoiBI. After 6 months, only 'Butterfly' from the dodder experimen remained positive, whereas all other plants were negative, including the two plants from the grafting experiment that previously had tested positive. It is not known why PoiBI was lost from these two plants. Of $\approx 200$ attempts, only the one from the dodder experiment was successful. The reason for the low efficiency of dodder transmission of PoiBI is not known, nor is it known why the grafting experiments were unsuccessful. However, we did not have a strong confirmation of the actual connection of vascular tissue between the donor and recipient, although the viability of scions for at leas 3 months indicated that a vascular connection had been established.

PCR products from the phytoplasma-infected 'Butterfly' were cloned and sequenced together with PCR products from poinsettia donor plants. Alignment of these sequences with the PoiBI 16S rRNA sequence (GenBank accession no. AF190223) confirmed the identity of these sequences to PoiBI 16S rRNA.

Cuttings (second generation) were taken from PoiBI-infected 'Butterfly', and later cuttings were taken from these (third generation). Cuttings from second- and third-generation plants were PCR-tested several times for the presence of PoiBI during the next 1.5 years and all were found to be positive, demonstrating that PoiBI is transmitted to and is viable in $E$. milii 'Butterfly'.

The third-generation 'Butterfly' cuttings (six cuttings) were compared to six noninfected 'Butterfly' cuttings for their branching phenotype and also compared for other phenotypic traits after 6 months of growth (Fig. 1). Uninfected plants had $3.3 \pm 1.9$ (X $\pm \mathrm{SE}, \mathrm{n}=6$ ) axial branches per plant, whereas infected plants had $14.8 \pm 1.7(\mathrm{X} \pm \mathrm{SE}, \mathrm{n}$ $=6$ ) axial branches per plant. A $t$ test was performed on the data with $t=11.1$ and $P<$ 0.0001 . Based on visual inspection, infected plants had smaller leaves and less vegetative growth than uninfected plants. Also, at the time of comparison, infected plants were devoid of flowers, whereas uninfected plants had a few flowers. These observations are unique to E. milii, as leaf size and flowering are not affected in poinsettia by phytoplasma infection, at least not using our growth conditions These observations strongly indicate that PoiB has a similar branching effect in E. milii as in 


\section{Plant Pathology}
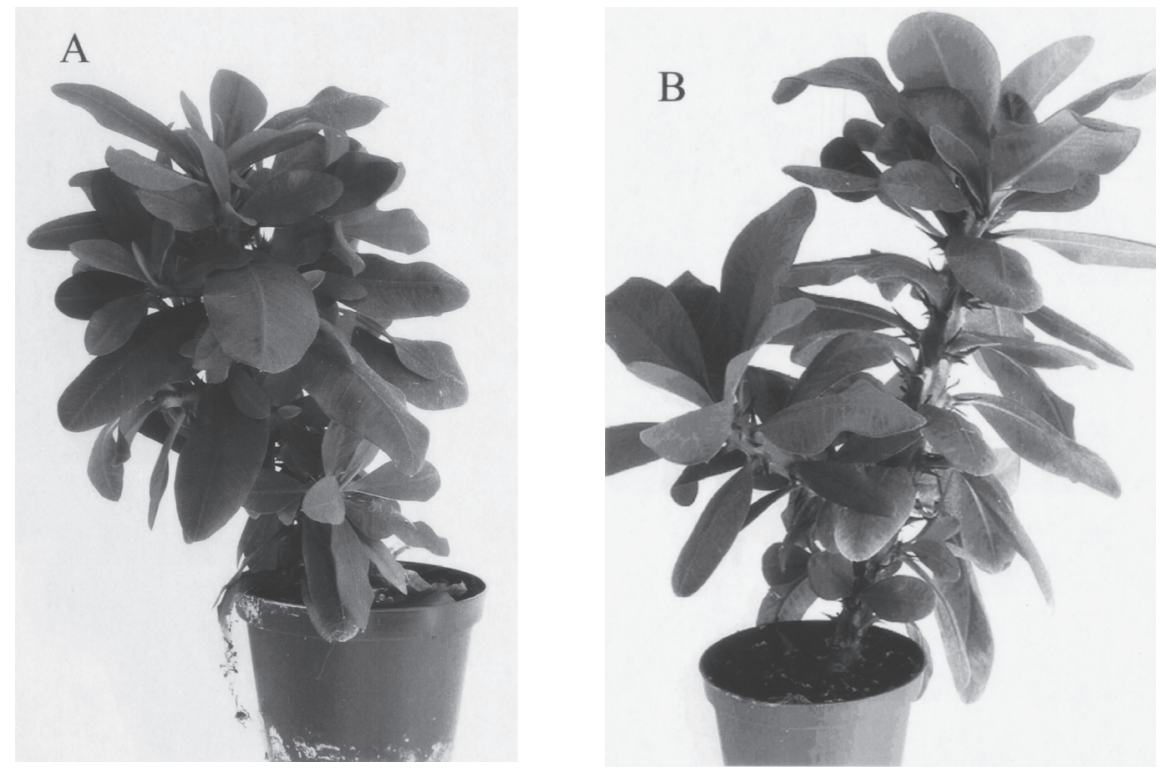

Fig. 1. E. milii infected with PoiBI (A) and uninfected (B).

poinsettia, although a test under production conditions remains to be done. It also remains to be determined whether other cultivars of $E$. milii are susceptible to PoiBI, but considering the broad susceptibility of poinsettia to PoiBI, it may be speculated that PoiBI will also infect a broad range of $E$. milii cultivars. In initial experiments, we found that $E$. milii/E. milii grafts are very easy to produce and establish. Therefore, a straightforward approach for transmitting PoiBI to other E. milii cultivars would be to use PoiBI infected 'Butterfly' as the donor in grafting experiments, which is equivalent to the method used commercially to infect poinsettias.

It has been shown that PoiBI has a broad host range in Euphorbiaceae (Preil and Ebbinghaus, 1998 and this study) and induces increased branching in these taxa. The next step would be to determine if PoiBI can infect taxa outside the Euphorbiaceae, and eventually, to investigate the effect on branching on such host plants. If possible, this may lead to significantly improved ornamental plants and eventually decrease the need for chemical growth regulation.

\section{Literature Cited}

Abad, J.A.,C. Randall, and J.W. Moyer. 1997. Factors influencing detection of phytoplasmas associated with Poinsettia. Phytopathology 87:S1

Bech, K. and K. Rasmussen. 1996. Euphorbia pulcherrima, methods to eliminate Poinsettia mosaic virus (PnMV) and reinfection by different methods to reveal the 'nature' of the branching factor. Acta Hort. 432:176-186.

Ecke, Jr., P., O.A. Matkin, and D.E. Hartley. 1990. The poinsettia manual. $3^{\text {rd }}$ ed. Paul Ecke Poinsettias, Encinitas, Calif.

Lee, I.M., D.E. Gundersen-Rindal, R.E. Davis, and I.M. Bartoszyk. 1998. Revised classification scheme of phytoplasmas based on RFLP analyses of 16S rRNA and ribosomal protein gene sequences. Intl. J. Syst. Bacteriol. 48:1153-1169.

Lee, I.M., M. Klopmeyer, I.M. Bartoszyk, D.E. Gundersen-Rindal, T.S. Chou, K.L. Thomson, and R. Eisenreich. 1997. Phytoplasma induced free-branching in commercial poinsettia cultivars. Nat. Biotechnol. 15:178-182.

Preil, W., and R. Ebbinghaus. 1998. Euphorbia fulgens als neue topfpflanze. Deutscher Gartenbau 4:22-23.

Zhu, H., F. Qu, and L.H. Zhu. 1993. Isolation of genomic DNAs from plants, fungi and bacteria using benzyl chloride. Nucleic Acids Res. 21:5279-5280. 